\title{
Effects of microbeam radiation therapy on normal and tumoral blood vessels
}

\author{
Audrey Bouchet ${ }^{\mathrm{a}, ~ *}$, Raphäel Serduc ${ }^{\mathrm{b}, \mathrm{c}}$, Jean Albert Laissue ${ }^{\mathrm{d}, 1}$, Valentin Djonov ${ }^{\mathrm{a}, 1}$ \\ ${ }^{a}$ Institute of Anatomy, University of Bern, Baltzerstrasse 2, CH-3000 Bern 9, Switzerland \\ b INSERM, U836, Grenoble F-38043, France \\ ${ }^{\mathrm{c}}$ Université Joseph Fourier, Grenoble Institut des Neurosciences, UMR-S836, Grenoble F-38043, France \\ d University of Bern, Hochschulstrasse 4, CH-3012 Bern, Switzerland
}

\section{A R T I C L E I N F O}

\section{Article history:}

Received 8 January 2015

Received in revised form

22 April 2015

Accepted 25 April 2015

Available online 23 May 2015

\section{Keywords:}

Microbeam radiation therapy

Healthy tissue

Tumor

Vessels

\begin{abstract}
A B S T R A C T
Microbeam radiation therapy (MRT) is a new form of preclinical radiotherapy using quasi-parallel arrays of synchrotron X-ray microbeams. While the deposition of several hundred Grays in the microbeam paths, the normal brain tissues presents a high tolerance which is accompanied by the permanence of apparently normal vessels. Conversely, the efficiency of MRT on tumor growth control is thought to be related to a preferential damaging of tumor blood vessels.

The high resistance of the healthy vascular network was demonstrated in different animal models by in vivo biphoton microscopy, magnetic resonance imaging, and histological studies. While a transient increase in permeability was shown, the structure of the vessels remained intact. The use of a chick chorioallantoic membrane at different stages of development showed that the damages induced by microbeams depend on vessel maturation. In vivo and ultrastructural observations showed negligible effects of microbeams on the mature vasculature at late stages of development; nevertheless a complete destruction of the immature capillary plexus was found in the microbeam paths. The use of MRT in rodent models revealed a preferential effect on tumor vessels. Although no major modification was observed in the vasculature of normal brain tissue, tumors showed a denudation of capillaries accompanied by transient increased permeability followed by reduced tumor perfusion and finally, a decrease in number of tumor vessels. Thus, MRT is a very promising treatment strategy with pronounced tumor control effects most likely based on the anti-vascular effects of MRT.
\end{abstract}

(C) 2015 Published by Elsevier Ltd on behalf of Associazione Italiana di Fisica Medica. This is an open access article under the CC BY-NC-ND license (http://creativecommons.org/licenses/by-nc-nd/4.0/).

\section{Background}

Since the 1990s, microbeam radiation therapy (MRT) has been proposed and developed as a new form of preclinical radiotherapy [1-6]. MRT, invented at the National Synchrotron Light Source (NSLS) of Brookhaven National Laboratory (BNL), Upton, New York, uses highly collimated, quasi-parallel arrays of X-ray microbeams with an energy of 50-600 keV. This can now be produced by third generation synchrotron sources such as the European Synchrotron Radiation Facility (ESRF), in Grenoble, France. The effects of microbeams derived from synchrotron X-rays was first studied in cerebral tissues, where arrays of microplanar beamlets safely

\footnotetext{
* Corresponding author.

E-mail address: audrey.m.bouchet@gmail.com (A. Bouchet).

1 Equal contributor.
}

delivered radiation doses to normal animal brains that were much higher than maximum doses tolerated by the brain of animals or patients from any standard millimeter-wide radiosurgical beam $[3,5]$. This unusually high normal tissue tolerance was accompanied by the permanence of apparently normal vessels in the path of the microbeams, with preserved endothelial nuclei, while neuronal and astrocyte nuclei had disappeared [3]. This resistance of normal vascular tissue was viewed as a central feature for the rationale of planning the use of MRT for brain tumors [5]. Studies performed over the past decades have proved the therapeutic efficiency of MRT for different types of tumors. Further, the tolerance of normal tissues to this type of irradiation has been characterized in several animal models. Uni- or bidirectional MRT reduced or even stopped the growth of tumors such as the EMT6 murine mammary carcinoma [7], an aggressive mouse model of squamous cell carcinoma [8], and of 9L intracerebral gliosarcoma in rats [3,9-12]. The tolerance of normal tissues to microbeam irradiation (MBI) was 
surprisingly high in the rat skin [13], fast-growing immature tissues such as the duck brain in ovo [14], the chick chorioallantoic membrane (CAM) [15] and, the in vivo cerebella of normal, suckling Sprague-Dawley rat pups [16], as well as adult rat and mouse brains $[3,5,17,18]$ and in normal weanling piglet cerebella $[19,20]$ (Fig. 1A). Normal tissues, even when developing, were deemed to be about 10 times more tolerant of microbeam irradiation than conventional, broad beam (BB), exposure. For instance, an $11 \mathrm{~mm}$ wide microbeam irradiation of the spinal cord did not induce any paralysis for entrance doses up to $500 \mathrm{~Gy}$ while all the rats exposed to entrance doses $>150$ Gy delivered by a $1.3 \mathrm{~mm}$ wide single beam rapidly developed paralysis [21]. An example of a histological section of the hindbrain of a weaned piglet is shown in Fig. 1A. Microbeams can selectively ablate neurons, oligodendrocytes and astrocytes in the central nervous system in their path, without causing tissue necrosis. This surprising tolerance of normal brain tissue might be a consequence of the radioresistance of normal brain vasculature. Conversely, it has been proposed that the efficiency of MRT on tumor growth control is related to a preferential damaging of tumor vessels.

During the last ten years, the response of normal and tumor vessels to spatially fractionated high dose exposures has been studied extensively. In the present report we attempt to present a summary of the current knowledge and progress in this area.

\section{Normal vessel response to microbeam radiation}

When applied to the brain of rodents, microbeam irradiation does not modify any of the numerous structural or functional parameters such as blood volume, vascular density and perfusion, even after delivery of doses up to $1000 \mathrm{~Gy}[17,18]$. The endothelial cell lining of the vessels in the microbeam paths remains intact, whatever the delay of observation (from a few hours up to 1 month after irradiation by microbeams (Fig. 1B)); no denudation of the vascular basement membrane, no hemorrhages, no thrombi have been observed. A transient diffusion of sulforhodamine B (SRB, $0.58 \mathrm{kDa}$ ) was observed by intravital two photon microscopy in microbeam irradiated regions from $12 \mathrm{~h}$ until 12 days after irradiation, but only for a high dose exposure (1000 Gy), not for a moderate dose (312 Gy) [17]. The perivascular deposition of SRB has been observed within the microbeams path but not in the surrounding tissue, indicating that irradiation by microbeams enhances the vascular permeability. Brain edema, usually observed after conventional radiotherapy, is considerably reduced after MRT. Edema of a mixed, i.e. vasogenic and cellular, type has been observed using magnetic resonance (MR) diffusion tensor imaging at an early stage $(12 \mathrm{~h})$ after exposure to very high peak entrance dose (1000 Gy), while a moderate and clinically relevant microbeam irradiation (e.g., $312 \mathrm{~Gy}$ ) only induced a short and transient cellular edema, but no blood brain barrier breakdown (BBBB) [18]. These results have been confirmed by water content measurement in brain tissues [18]. Indeed, there was an increase in cerebral water content (CWC) the first week after exposure, while no BBBB and no changes in brain vessel permeability nor in CWC were measured after exposure to a lower dose of $312 \mathrm{~Gy}$.

Whatever the dose delivered, brain vessels remained perfused and no changes in animal behavior were observed [20]. These results are concordant with those described by Dilmanian et al. (2003) [22], who showed that the BBBB for albumin-FITC took place in only $9 \mathrm{~L}$ gliosarcoma tumor vessels, which were not permeable before MRT, and not in capillaries in normal brain tissue after MBI (entrance dose $800 \mathrm{~Gy}$, microplanar width $27 \mu \mathrm{m}$, microplanar spacing $200 \mu \mathrm{m}$ ) [22]. Brain vessel vulnerability to irradiation has been shown to change with: 1) radiation dose (see above); 2) microbeam width; and 3) vessel size. In 2009, Serduc et al. demonstrated that at constant valley doses (dose received between the microbeam paths), high radiation peak entrance doses ( $860 \mathrm{~Gy}$ ), delivered to the head of rats by two cross-fired arrays of thin microbeams $(25 \mu \mathrm{m})$, were more neurotoxic than lower doses (480 Gy) deposited by arrays of $50 \mu \mathrm{m}$ wide microbeams. Indeed, normal rats, exposed to such a hemi-cranial brain irradiation by two cross-fired arrays of $25 \mu \mathrm{m}$ or $50 \mu \mathrm{m}$ wide beamlets, but to the same valley dose, survived 4.5 days (median survival time, MST), or 68 days after MBI, respectively [23]. However, when the microbeam widths were increased up to $75 \mu \mathrm{m}$ and the radiation peak entrance dose reduced to $320 \mathrm{~Gy}$, MST of rats decreased to 48 days. This demonstrated that the brain vascular network can tolerate $50 \mu \mathrm{m}$ wide lesions induced by microbeams, but not with peak doses exceeding $500 \mathrm{~Gy}$. This is important when minibeam radiation therapy, i.e., using beamlets with a size range of $>100-900 \mu \mathrm{m}$, is applied to brain tissues using hectogray doses. However, until now, no work has been published on the tolerance of normal brain vessels to such wide beams. Preliminary unpublished data obtained in INSERM U836 laboratories tend to show that irradiation by

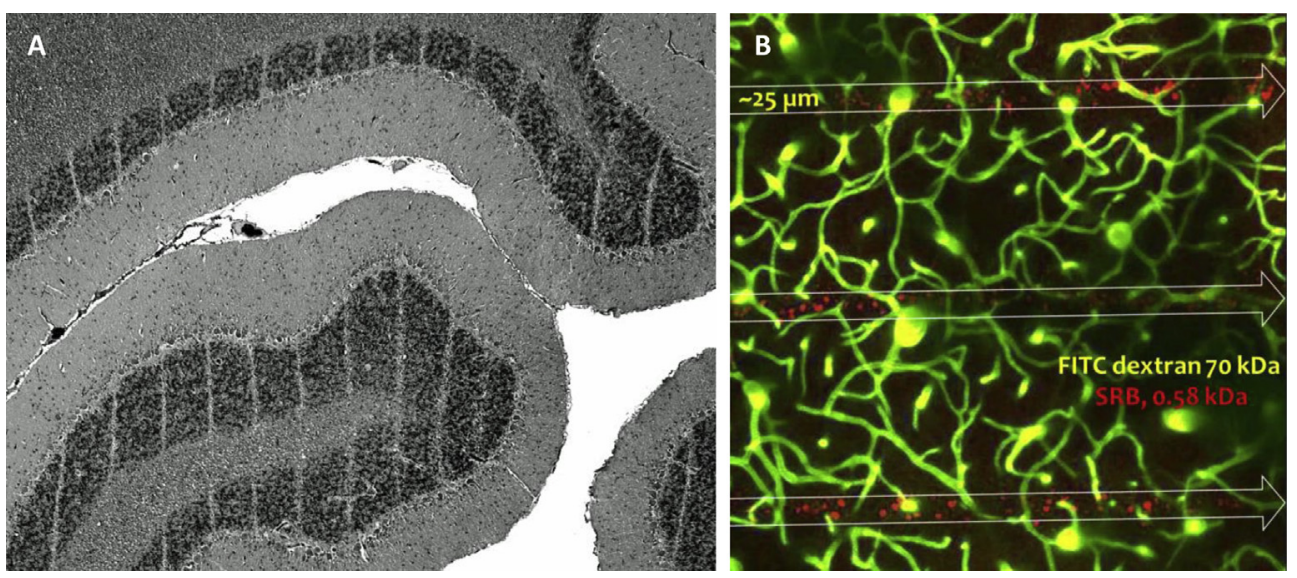

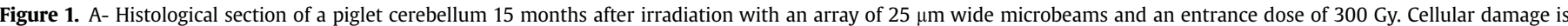

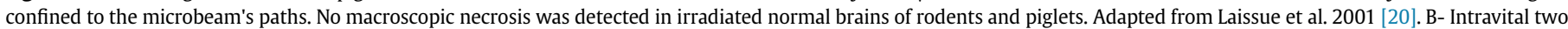

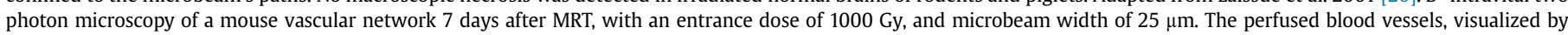

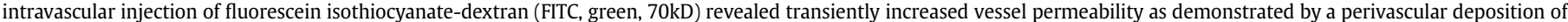

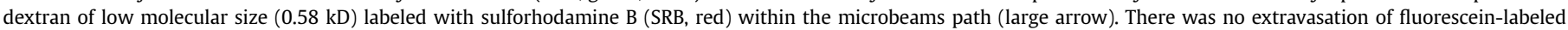

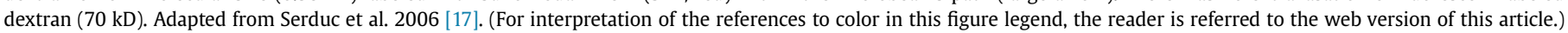


beamlets wider than $100 \mu \mathrm{m}$ provokes vessel responses that are in marked contrast with the quick repair observed after irradiation by thin microbeams. For example, $200 \mu \mathrm{m}$ wide beams induced macroscopic brain necrosis due to angionecrosis, beginning 3 weeks after exposure to an entrance dose of $400 \mathrm{~Gy}$. Studies of the influence of beam size and dose on brain vessels are currently in progress, but need further expansion to determine whether the demonstrated vessel resistance to MBI also occurs after exposure to minibeams. Finally, large vessels display an extraordinary tolerance to MBI at least in skeletal muscles. Van der Sanden et al. irradiated saphenous arteries in the lower limbs of mice with peak entrance doses up to $1000 \mathrm{~Gy}$, delivered by an array of $50 \mu \mathrm{m}$ wide microbeams spaced $400 \mu \mathrm{m}$ on center [24]. The microscopically narrow arterial smooth muscle cell layer segments in the microplanar beam paths became atrophic and fibrotic in a dose-dependent pattern. The wide tunica media segments sited between those paths hypertrophied but the arteries remained patent and perfused, as observed in situ by two-photon microscopy, as well as histopathologically. Comparable doses delivered by un-segmented broad beams destroy normal arteries [25]. This vascular tolerance is instrumental in maintaining vascular oxygen and nutrient supply and is a major advantage of MRT. The tolerance relates to the high therapeutic ratio of MRT for solid tumors in rodent brains. The stability of the normal vasculature exposed to MRT is in marked contrast to the changes that occur after conventional radiotherapy.

\section{Microbeam radiation-induced damage depends on the vascular maturation stage}

The differential effect of microbeams on mature and immature vessels was also illustrated by in vivo and morphometric observations of a chick chorioallantoic membrane (CAM) model at different stages of development. A CAM is an almost pure avian vascular system in which immature vessels, lacking periendothelial coverage, mature within few days to vessels with such coverage [15]. CAMs cultivated in Petri dishes were irradiated with microplanar beams (width, $\sim 25 \mu \mathrm{m}$; interbeam spacing, $\sim 200 \mu \mathrm{m}$ ) at entrance doses of $200 \mathrm{~Gy}$. The in vivo monitoring of immature CAM vasculature over 8 days of development (CAM8) revealed a near total destruction of the immature capillary plexus $6 \mathrm{~h}$ after MBI with $200 \mathrm{~Gy}$ (Fig. 2a). Large areas were no longer perfused $6 \mathrm{~h}$ after irradiation. Some solitary capillaries sited in the path between the beams were partially preserved, with intact supplying vessels running parallel between the beams. Small arterioles and veins

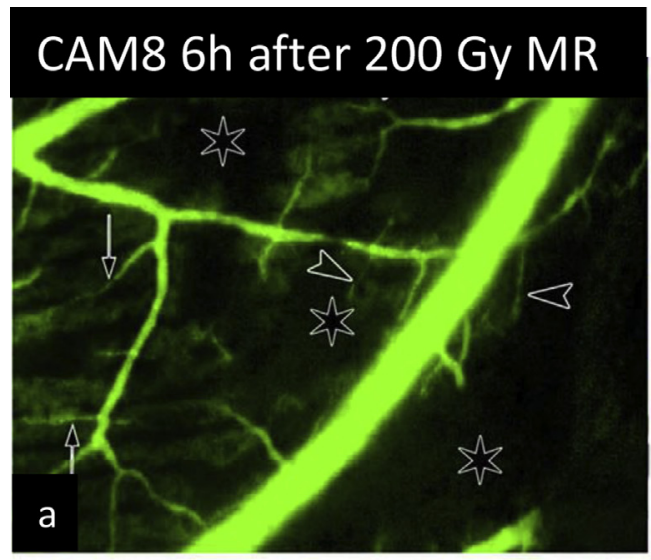

Relative density of perfused

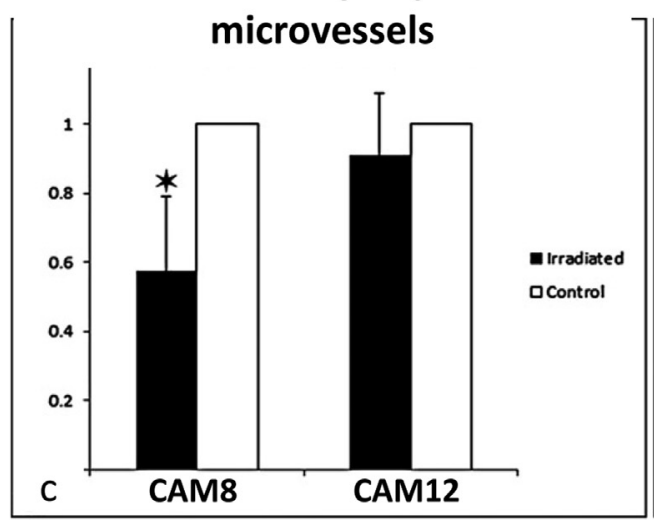

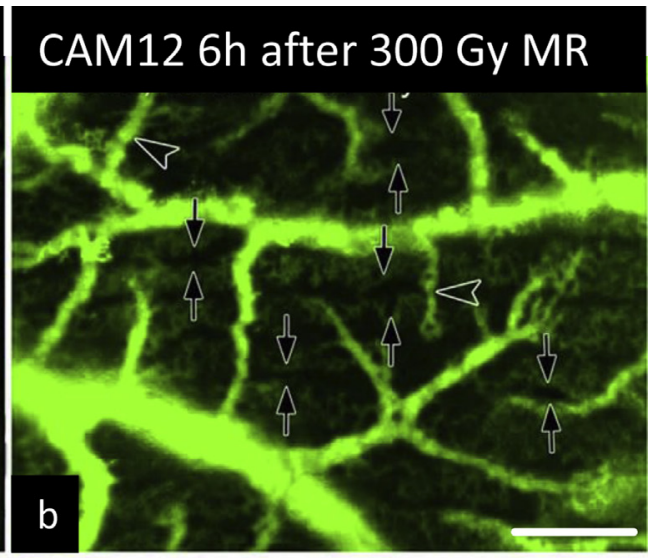

Fraction of unperfused microvessels in vivo

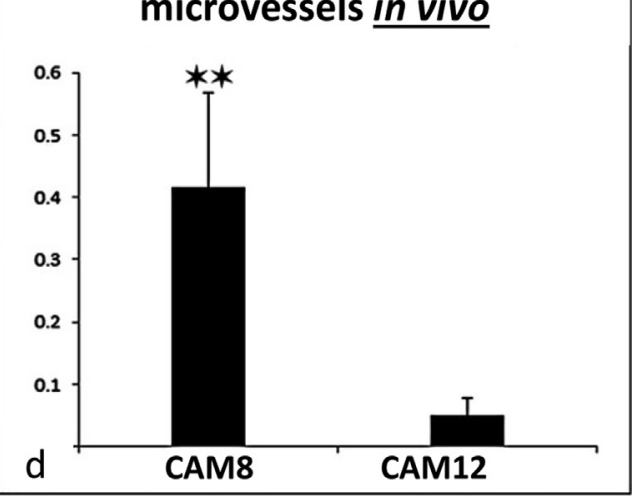

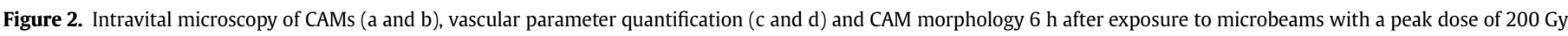

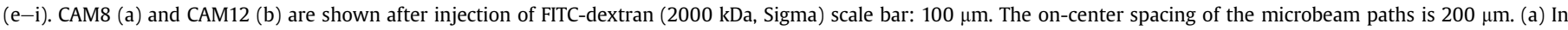

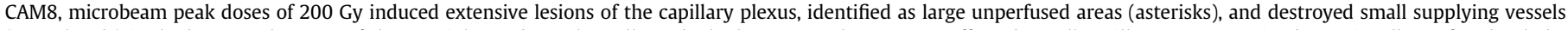

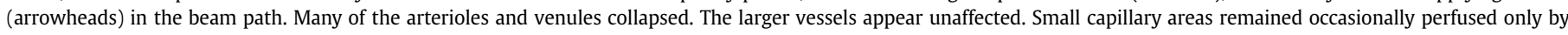

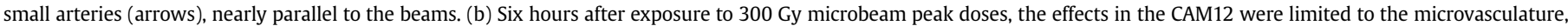

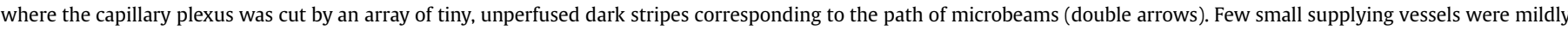

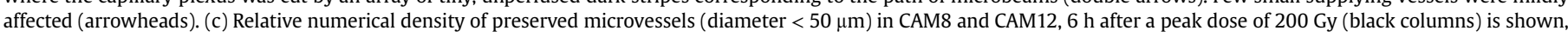

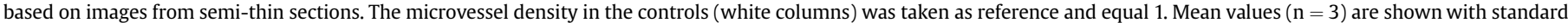

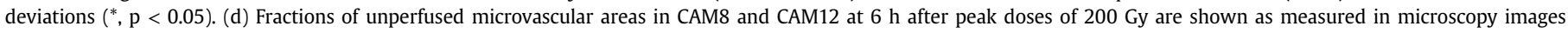
obtained in vivo. Mean values $(\mathrm{n}=4)$ and standard deviations $(* *, \mathrm{p}<0.001)$ are given. 
were collapsed or destroyed in the beam path, while larger arteries and veins were not affected by the irradiation.

Conversely, 200 Gy MBI slightly affected CAM mature microvasculature at 12 days of development (CAM12); negligible effects on the vasculature were observed. Relative numerical density of preserved microvessels $6 \mathrm{~h}$ after $200 \mathrm{~Gy}$ MBI was significantly $(\mathrm{p}<0.05$ ) higher in CAM12 than in CAM8 (Fig. $2 \mathrm{c}$ ). In addition to this, the fraction of unperfused microvascular areas was significantly ( $\mathrm{p}<0.001$ ) lower in CAM12 than in CAM8 (Fig. 2d) $6 \mathrm{~h}$ after $200 \mathrm{~Gy}$ MBI. Even when the dose was increased to $300 \mathrm{~Gy}$ (Fig. 2b), the CAM12 capillary plexus was less damaged than that of CAM8 irradiated with a dose of $200 \mathrm{~Gy}$. Infact, in CAM12 the capillary vessels revealed only tiny, sharply demarcated, unperfused stripes along the beam path $6 \mathrm{~h}$ after exposure to $300 \mathrm{~Gy}$ (Fig. 2b). Small supplying vessels were barely affected. The CAM vasculature recovered rapidly and only small defects were detectable in the capillary plexus $24 \mathrm{~h}$ after $300 \mathrm{~Gy}$ MBI. The vascular pattern appeared similar to that of the non-irradiated CAMs [15]. Microscopic and ultrastructural observations showed a destruction of the plexus of immature capillaries in the irradiated zone while the capillaries were preserved in between (Fig. 3a-e).

This comparison of MBI effects on different maturation stages of the avian vessel network has clearly shown that vascular toxicity and physiological effects of microbeam radiation depend on the stage of capillary maturation. The time- and dose-dependent MBIinduced vascular changes in CAMs differ markedly from those seen in adult mice. It is not surprising that CAM vasculature, a transient, developmental organ, should be more vulnerable to this type of irradiation than the highly differentiated vasculature of adult organisms.

\section{Microbeam radiation preferentially affects tumor vasculature}

Beyond the difference of microbeam effects on mature versus immature vessels, a preferential effect on tumor vessels was also described for different tumor types. The effect of MRT, with or without drug combination, on the enhancement of the survival of tumor bearing animals and on tumor growth control has been extensively described [3,7-12,26-28].

\section{Mammary carcinoma}

The therapeutic potential of different doses (75 Gy or $150 \mathrm{~Gy}$ ) deposited by $50 \mu \mathrm{m}$ wide microbeams or $500 \mu \mathrm{m}$ wide minibeams was tested on mouse mammary carcinomas (4T1), a moderately hypoxic tumor, resistant to radiotherapy and with a propensity to metastasize [27]. A peak entrance dose of $150 \mathrm{~Gy}$ administered in arrays of $50 \mu \mathrm{m}$ wide beams was the most effective in delaying tumor growth. The $500 \mu \mathrm{m}$ beams at $75 \mathrm{~Gy}$ were unable to alter tumor growth compared to untreated tumors. The two other configurations gave intermediate tumor growth control. Administration of adjuvant anginex therapy (a novel anti-angiogenic peptide) to these different conditions of irradiation further increased the tumor growth delay.

Quantification of immunohistochemically stained endothelial cells (CD31) indicated that vessel density was significantly lower 14 days after irradiation in all treated groups in comparison to animals

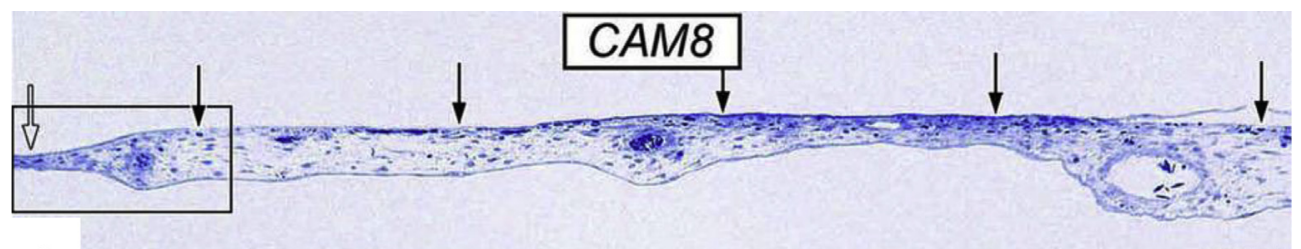

a

$300 \mu \mathrm{m}$
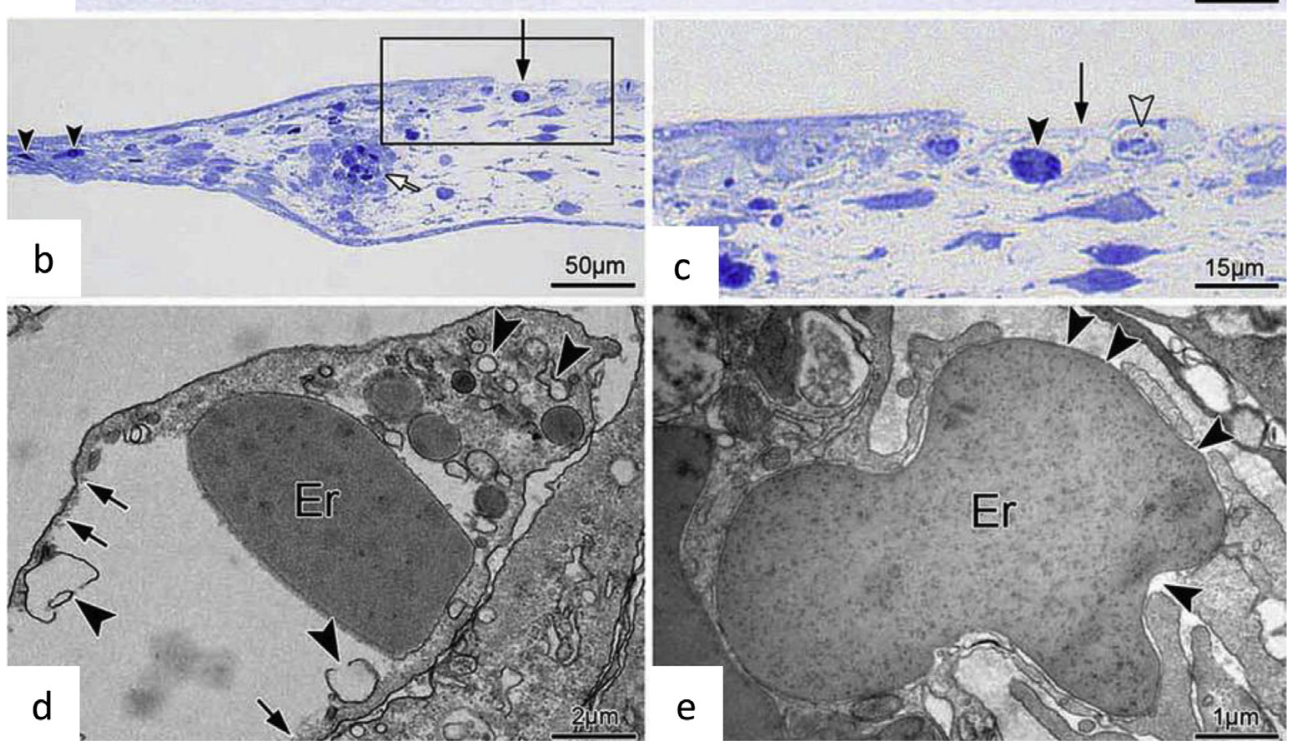

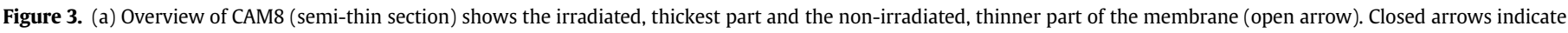

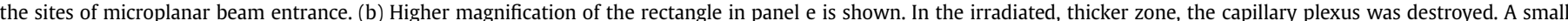

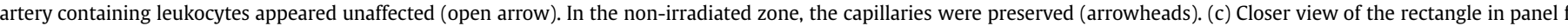

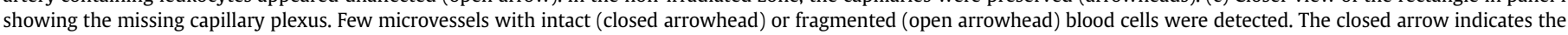

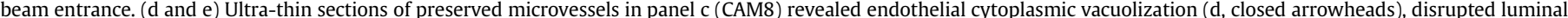

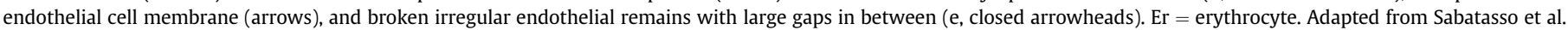
2011 [15]. 
in the untreated group [27]. The number of pericytes, stained by an anti-smooth muscle antibody ( $\alpha \mathrm{SMA}$; smooth muscle pericyteassociated blood vessels) significantly increased in the irradiated groups on day 14 after irradiation and seemed to correspond to a vascular normalization. Griffin and his colleagues proposed that this effect might be exploited to improve tumor control using agents targeting angiogenesis. A normalization of tumor vessels after MRT seems to be in opposition with the destruction of immature vessels observed on CAM and different tumor models. It is difficult to interpret and compare these results of normalization with the others studies of vascularization because of the markedly different conditions for the experiments. For example the different doses (Griffin et al. 2012: 75 or $150 \mathrm{~Gy}$ for 50 micron wide beamlets, 200 micron on center distance; Bouchet et al. 2010: $2 \times 400 \mathrm{~Gy}, 50$ micron wide beamlets spaced at 200 micron on center) may have played an important role also for MRT, as single doses of 5-10 Gy (SRS/SBRT) cause relatively mild vascular damage, whereas higher doses result in severe vascular damage and reduced blood perfusion [29]. Also, the publication of Griffin et al. excepted, there are extremely few published data available on the presence or absence of pericytes around remaining vessels of experimental tumors, and on their influence on endothelial cells at the moment of irradiation. Further, the tumor vasculatures are as diverse as are the different tumor types and may thus react to radiation in many different modes.

In tumors treated with minibeams delivering $75 \mathrm{~Gy}$, the presence of areas reactive for pimonidazole, a marker of hypoxia, did not differ from the mean pimonidazole staining intensity in control tumors [27]. Conversely, tumors treated with microbeams (150 Gy) revealed a substantial reduction of overall pimonidazole staining intensity 1-14 days after MRT. The authors interpreted this diminution as an evidence of a decrease in tumor hypoxia after exposure to 150 Gy (MRT). However, only a limited number of tumors have been analyzed and statistical analysis concerning the effect of hypoxia is lacking. We need also keep in mind that pimonidazole, injected intravenously, might not have been delivered to unperfused tissue areas after the MRT. This may potentially be the reason for the false negative results.

Administration of the adjuvant Anginex further exacerbated the vascular modifications induced by MRT in all combination treatment groups and illustrated the potential of an antiangiogenic therapy coupled with MRT.

\section{Brain tumors}

MRT efficiently slows down the intracranial 9L gliosarcoma growth in rats (Fig. 4A and B). In addition, an in vivo MR imaging follow-up has demonstrated a significant decrease in the fractional blood volume in the tumor, and in diameter of tumor vessels, with cross-fired arrays beginning at 8 days after MRT [26]. Simultaneously, a striking remodeling of the tumor vasculature was detected histologically, as evidenced by a decreased density of tumor vessels with increased inter-vessel distances and endothelial denudation $[26,30]$ (Fig. 5A-C). These changes were associated with a significant decrease of the local tissue saturation with oxygen, monitored by magnetic resonance based measurement $\left(\mathrm{MR} \_\mathrm{SO}_{2}\right)$ and with an histologic over-expression of an indirect marker of hypoxia (GLUT1) in tumor tissue [30] (Fig. 5D-F). Taken together, these results indicated that MRT-induced tumor vessel damage led to a reduction of tumor oxygenation a few days after MRT. Normal blood vessels were damaged only in peritumoral brain tissue areas irradiated by both intersecting arrays. Thus, during the subacute phase ( 6 weeks post MRT), brain edema and overexpression of vascular endothelial growth factor were observed in such areas subjected to cross-fire beams. Conversely, vascular parameters, vessel morphology and oxygenation were not or only slightly modified in normal brain regions exposed to one of the two MRT arrays. As reviewed above, vasculature of the normal brain exposed to MRT remained sufficiently perfused to prevent hypoxia in the normal brain. These data showed for the first time that MRT preferentially damages tumor vessels, but does not impair normal brain blood vessels in a rat bearing an intracerebral 9L gliosarcoma.

The blood-brain barrier permeability (as indicated by contrast MR enhancement, Fig. 6) did not change in normal brain tissues after MRT crossed by one or two directions of microbeam arrays [26]. Conversely, MRT led to an increase in tumor vessel permeability 2 and 5 days after irradiation, when the MR signal enhancement reached 23.9 and $26.6 \%$, respectively, whereas in non-irradiated tumors the enhancement was only 16.6 and $20.3 \%$ ( $p<0.001$ ). Thus, compared to the intrinsic permeability of untreated tumor vessels, the tumor vessel permeability was higher on day 2 and day 5 after irradiation, by 44 and 31\%, respectively. This transient breakdown of the tumor-blood barrier might be used to deliver intravenously injected drugs to the tumor core, thus enhancing drug supply and therapeutic efficiency [26]. From the fifth to the fifteenth day after irradiation the blood vessel permeability of irradiated tumors decreased by 74\%, ( $p<0.001$ ). We interpret this as an impaired supply of contrast agent to the tumor which correlates with the significant decrease in tumor blood volume and in number of tumor vessels that we observed at the same delays after MRT (Fig. 5B, C).

\section{MRT versus broad beam irradiation}

There are several probable reasons why MRT gives a higher therapeutic index for tumors than broad beam irradiation. Microbeams produce steep dose gradients between tissue slices receiving

\section{GLS brain tumor growth control and increase in survival}
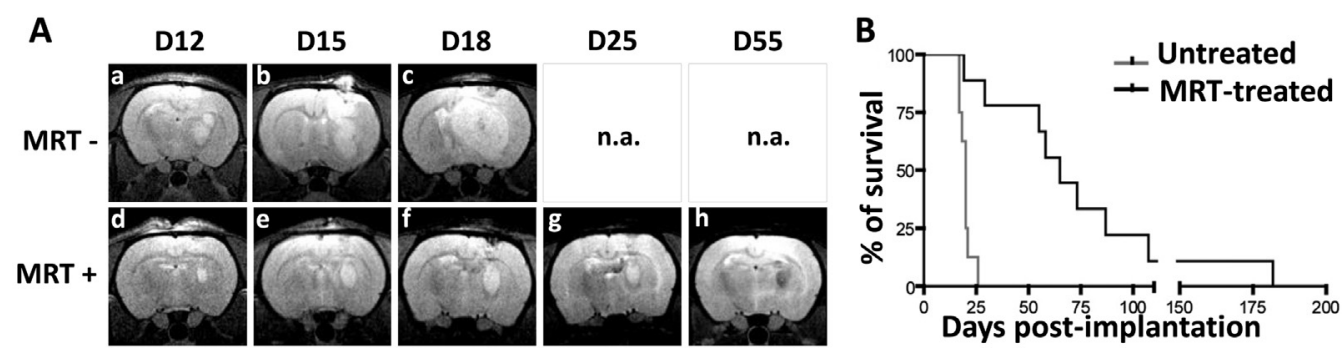

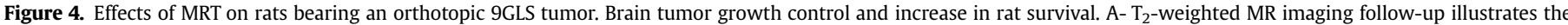

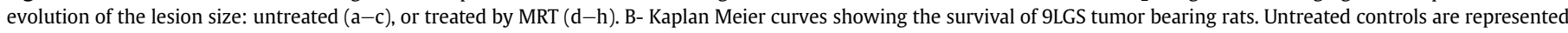

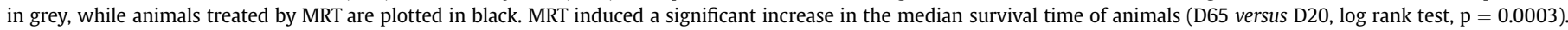




\section{Decrease in 9GLS tumor blood volume, vessel density and endothelial denudation}

A
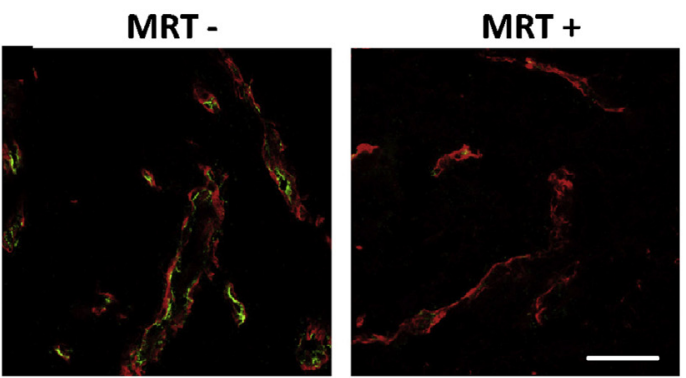

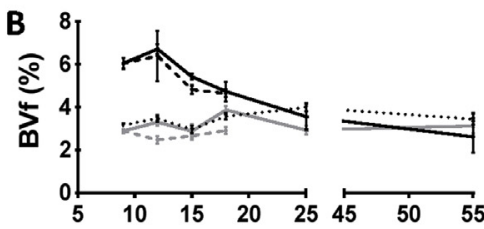

C

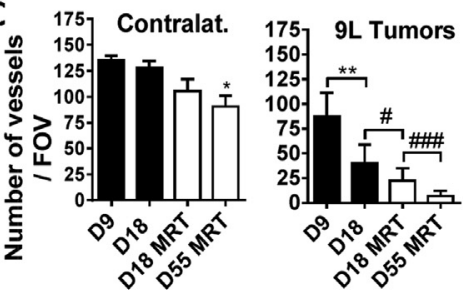

\section{Decrease in 9GLS tumor oxygenation and over- expression of hypoxia marker}

D

MRT -
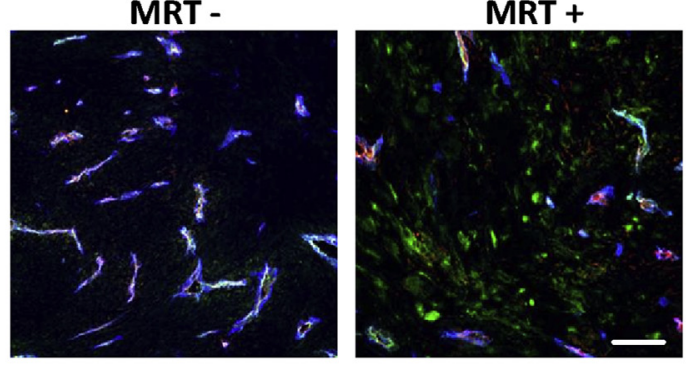

E

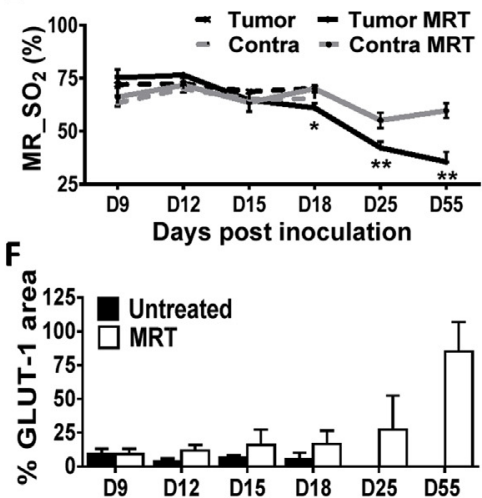

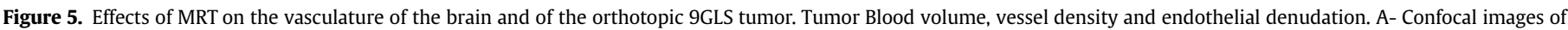

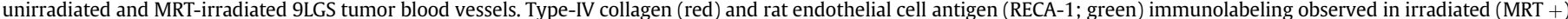

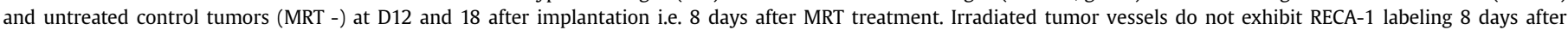

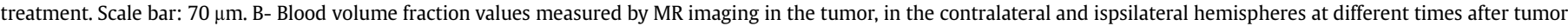

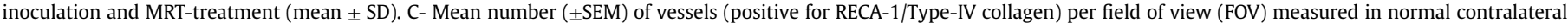

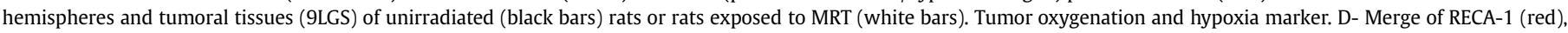

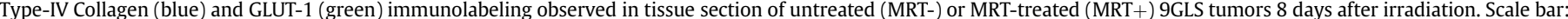

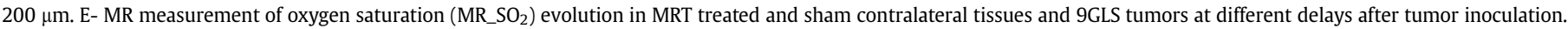

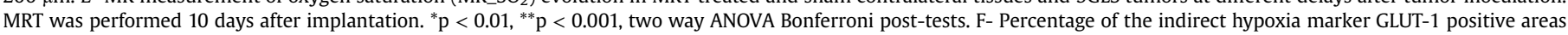

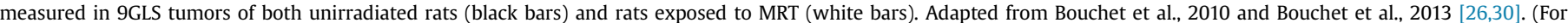
interpretation of the references to color in this figure legend, the reader is referred to the web version of this article.)

the peak and valley doses: they have a $90 \%-10 \%$ dose fall-off, about 200 times steeper than that of a Gamma Knife. The radiotoxic dose is therefore confined to a very narrow zone while the integrity and functionality of adjacent normal tissue in the valleys between the

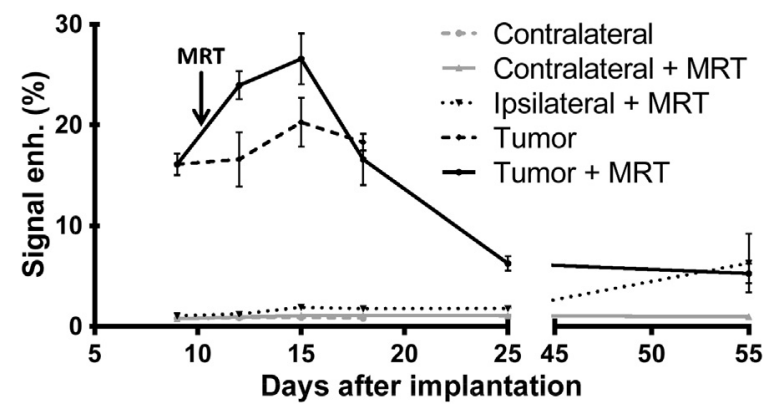

Figure 6. Vessel permeability measurements, indicated as contrast MR signal enhancement. High vessel permeability values measured by MR imaging in the intracranial tumor (9GLS), versus those noted in normal parts of the contralateral and ispsilateral brain hemispheres, at different times after tumor inoculation and MRT (mean \pm SD). Adapted from Bouchet et al. 2010 [26]. peaks is barely damaged. Further, spatial fractionation results in a large specific contact surface between peak and valley zones, much larger than that provided by a single broad beam [31]. This extended contact surface is instrumental for the repair of heavily irradiated tissues in peak regions by much less damaged tissues in the valleys.

The important role of the high tolerance of the normal microvasculature and arteries to irradiation by microbeams and the tumor vasculature of 9LGS, for instance, has been described here; they may result in tumor hypoxia and shrinkage. Conversely, normal brain tissues exposed to microbeams remain sufficiently perfused and maintain normoxia. Irradiation of normal rat brains with microbeams is known to elicit proteomic responses that are indicative of oncogenesis as well as in proteomic changes associated with bystander effects, indicative of apoptosis mediated by reactive oxygen species. The acid-sphingomyelase mediated endothelial apoptosis suggested after radiation-induced DNA damage might be relevant in case of MRT as well [32]. Further, potentially anti-oncogenic apoptotic proteomic changes indicate that the collective interaction of such MB irradiation-induced bystander effect proteins might confer a protective effect on normal tissues [33]. 
Transcriptional gene expression analysis of intracerebral gliosarcomas in rats revealed that MRT induced a response specific of tumor tissue in comparison to normal tissue [34]. The specific response of the tumor tissues is composed by 316 significantly modulated transcripts which are mainly related to immune/inflammatory response, as described in a previous study [35]. But there are other early biological processes underlying the differential effect of MRT on tumor and normal brain [34]. Among the genes isolated, 30 were not detected by microarray in normal brain tissues, neither before nor after MRT. Polo-like kinase 1 (Plk1) and some associated genes (as Ccnb1, Cdc20, Pttg1), known to be necessary for efficient cell division and proposed as potential therapeutic target in human tumors [36], showed a decreased expression after MRT. A cluster of 20 genes presented a similar evolution of expression during the first days/weeks after MRT and their inhibition suggests that a massive disorganization of cell division takes place. In addition, a progressive numerical increase in giant/polyploid cells and in the proportion of polyploid cells among mitotic cells beginning 2 days after MRT might be associated with mitotic cell death. This is in agreement with an early cytotoxic effect on tumor cells previously described for the same tumor model [26,37].

On the contrary Areg, thought to be involved in tumor escape and radioresistance [38], was significantly overexpressed in 9L gliosarcoma after MRT. This gene is an activator of the epidermal growth factor receptor (EGFR) resulting in processes that are critical for tumor survival and progression, involving tumor cell proliferation, invasiveness, motility, angiogenesis and inhibition of apoptosis. These potential effects point to Areg/Amphiregulin as potential target for increasing the therapeutic index of MRT. The up-regulation of Areg expression may indicate the emergence of survival processes in tumor cells triggered by the irradiation; while the modulation of the "Plk1 cluster genes", which relates to cytokinetic features of the tumor observed histologically after MRT, may partially explain the control of tumor growth by MRT. A comparison of the 9L brain tumor response after MRT or after synchrotron broad beam irradiation is currently under analysis. However, in an other model, Sprung et al. have identified MRT-induced immunity related modulations in EM6.5 breast tumors in mice that were clearly different from transcriptional changes induced by unsegmented broad beam X-rays [39].

\section{Discussion and perspectives}

Since the beginning and during the development of irradiation using synchrotron-generated X-ray microbeams, the hypothesis has emerged that the sparing of normal tissues was due to the resistance of normal blood vessels to microbeam irradiation, whereas immature tumor vessels cannot repair damage induced by MRT. Over the past decades, different techniques and approaches have tested and now lend support to this hypothesis. While no major modification was observed in normal vasculature $[17,18,26]$, tumors showed a denudation of the tumor vascular endothelium, a decrease in number of vessels $[26,27,30]$ leading to a decrease in perfusion [26] and to tumor hypoxia [30]. On the contrary, in another model study [27] the tumor vasculature presented signs of normalization of the vasculature (i.e. presence of pericytes) along with an improvement in the tumor oxygenation. However, these phenomena were studied in only a few tumor models. The consequences of tumor vessels modifications appear sometimes to be contradictory, in particular for changes in tumor oxygenation as observed in a brain and a mammary tumor [27,30]. Complementary studies with new approaches, such as in vivo stereotaxic microscopy, testing different tumor models, as well as larger animals such as pigs or pet animals are necessary for a better comprehension of vascular responses to MRT in normal tissues and tumors.
Although microbeam radiation preferentially affects tumor vessels in many ways, it would be simplistic to restrict the effects of MRT to vascular effects alone. In the light of recent studies other mechanisms seem to participate in the effectiveness of MRT. The mode of action of microbeams, as of any radiation therapy, should be considered as the sum of multi-level interactions of a complex nature. Indeed, cytotoxic effects on tumor cells have been observed [9], even before vascular parameters appeared to be modified [26], and the redistribution of the cell cycle in the tumor was shown to have occurred only a few hours after MRT [34]. MRT may also induce transcriptomic changes such as an overexpression of the resistance factor Areg which is critical for tumor survival and progression, as it involves tumor cell proliferation, invasiveness, motility, inhibition of apoptosis and angiogenesis [40]. Moreover, a strong involvement of immune and/or inflammatory responses after MRT has been revealed in several recent studies [34,35,39]. MRT effects on vasculature, tumor cell proliferation arrest and death, involvement of resistance mechanisms and immune response can all be studied separately but an integrated consideration of these mechanisms is now needed to understand the efficiency of MRT and to further increase the therapeutic potential of this promising method.

\section{Acknowledgments}

The authors are supported by the COST-Action TD1205.

The author thanks the Région Rhône-Alpes - Cible 2012, Ligue contre le cancer, Association pour la recherche contre le cancer, the ANR-11-LABX-0063/ ANR-11-IDEX-0007 and the Bernische Krebsliga.

\section{References}

[1] Brauer-Krisch E, Serduc R, Siegbahn EA, Le Duc G, Prezado Y, Bravin A, et al Effects of pulsed, spatially fractionated, microscopic synchrotron X-ray beams on normal and tumoral brain tissue. Mutat Res 2010;704:160-6.

[2] Slatkin DN, Dilmanian FA, Nawrocky MM, Spanne P, Gebbers JO, Archer DW et al. Design of a multislit, variable width collimator for microplanar beam radiotherapy. Rev Sci Instrum 1995;66:1459-60.

[3] Laissue JA, Geiser G, Spanne PO, Dilmanian FA, Gebbers JO, Geiser M, et al Neuropathology of ablation of rat gliosarcomas and contiguous brain tissues using a microplanar beam of synchrotron-wiggler-generated $\mathrm{X}$ rays. Int J Cancer 1998;78:654-60.

[4] Slatkin DN, Dilmanian FA, Spanne P. Method for microbeam radiation therapy. 1994.

[5] Slatkin DN, Spanne P, Dilmanian FA, Gebbers JO, Laissue JA. Subacute neuropathological effects of microplanar beams of x-rays from a synchrotron wiggler. Proc Natl Acad Sci U S A 1995;92:8783-7.

[6] Slatkin DN, Spanne P, Dilmanian FA, Sandborg M. Microbeam radiation therapy. Med Phys 1992;19:1395-400.

[7] Dilmanian FA, Morris GM, Zhong N, Bacarian T, Hainfeld JF, Kalef-Ezra J, et al. Murine EMT-6 carcinoma: high therapeutic efficacy of microbeam radiation therapy. Radiat Res 2003;159:632-41.

[8] Miura M, Blattmann H, Bräuer-Krisch E, Bravin A, Hanson AL, Nawrocky MM, et al. Radiosurgical palliation of aggressive murine SCCVII squamous cell carcinomas using synchrotron-generated X-ray microbeams. Br J Radiol 2006:79:71-5.

[9] Bouchet A, Boumendjel A, Khalil E, Serduc R, Brauer E, Siegbahn EA, et al. Chalcone JAI-51 improves efficacy of synchrotron microbeam radiation therapy of brain tumors. J Synchrotron Radiat 2012;19:478-82.

[10] Dilmanian FA, Button TM, Le Duc G, Zhong N, Pena LA, Smith JA, et al Response of rat intracranial 9L gliosarcoma to microbeam radiation therapy. Neuro Oncol 2002;4:26-38.

[11] Régnard P, Bräuer-Krisch E, Troprès I, Keyriläinen J, Bravin A, Le Duc G. Enhancement of survival of 9L gliosarcoma bearing rats following intracerebral delivery of drugs in combination with microbeam radiation therapy. Eur J Radiol 2008;68:S151-5.

[12] Regnard P, Duc GL, Bräuer-Krisch E, Tropres I, Siegbahn EA, Kusak A, et al Irradiation of intracerebral 91 gliosarcoma by a single array of microplanar xray beams from a synchrotron: balance between curing and sparing. Phys Med Biol 2008:53:861-8.

[13] Zhong N, Morris GM, Bacarian T, Rosen EM, Dilmanian FA. Response of rat skin to high-dose unidirectional X-ray microbeams: a histological study. Radiat Res 2003;160:133-42. 
[14] Dilmanian FA, Morris GM, Le Duc G, Huang X, Ren B, Bacarian T, et al. Response of avian embryonic brain to spatially segmented X-ray microbeams. Cell Mol Biol 2001;47:485-93.

[15] Sabatasso S, Laissue JA, Hlushchuk R, Graber W, Bravin A, Brauer-Krisch E, et al. Microbeam radiation-induced tissue damage depends on the stage of vascular maturation. Int J Radiat Oncol Biol Phys 2011;80:1522-32.

[16] Laissue JA, Lyubimova N, Wagner HP, Archer DW, Slatkin DN, Di Michiel M, et al. Microbeam radiation therapy. Proc SPIE 1999;3770:38-45. Denver, USA

[17] Serduc R, Verant P, Vial JC, Farion R, Rocas L, Remy C, et al. In vivo two-photon microscopy study of short-term effects of microbeam irradiation on normal mouse brain microvasculature. Int J Radiat Oncol Biol Phys 2006;64:1519-27.

[18] Serduc R, van de Looij Y, Francony G, Verdonck O, Van Der Sanden B, Laissue J, et al. Characterization and quantification of cerebral edema induced by synchrotron X-ray microbeam radiation therapy. Phys Med Biol 2008;53:1153-66.

[19] Laissue JA, Blattmann H, Wagner HP, Grotzer MA, Slatkin DN. Prospects for microbeam radiation therapy of brain tumours in children to reduce neurological sequelae. Dev Med Child Neurol 2007;49:577-81.

[20] Laissue JA, Blattmann H, Di Michiel M, Slatkin DN, Lyubimova N, Guzman R, et al. The weaning piglet cerebellum: a surrogate for tolerance to MRT (microbeam radiation therapy) in paediatric neuro-oncology. Proc SPIE 2001;4508:65-73. Washington.

[21] Laissue J. Preclinical microbeam radiation therapy (MRT): concepts experiments. 2003. Med. Appl. Work., Melbourne, Australia. Available from: http:// www.pathology.unibe.ch/Forsch.

[22] Dilmanian FA, Hainfeld JF, Kruse CA, Cool CD, Sze CI, Laterra JS, et al. Biological mechanisms underlying the X-ray microbeam effects of preferentially destroying tumors. Abstract No. Dilm0599. In: Corwin MA, Ehrlich SN, editors. Natl synchrotron light source act rep; 2002; 2003. http://www.bnl.gov/isd/ documents/25005/Abstracts/dilm0599.pdf.

[23] Serduc R, Bouchet A, Brauer-Krisch E, Laissue JA, Spiga J, Sarun S, et al. Synchrotron microbeam radiation therapy for rat brain tumor palliationinfluence of the microbeam width at constant valley dose. Phys Med Biol 2009;54:6711-24.

[24] Van der Sanden B, Bräuer-Krisch E, Siegbahn EA, Ricard C, Vial JC, Laissue JA Tolerance of arteries to microplanar X-ray beams. Print Int J Radiat Oncol Biol Phys 2010 Aug 1;77(5):1545-52.

[25] Nilsson A, Wennerstrand J, Leksell D, Backlund EO. Stereotactic gamma irradiation of basilar artery in cat. Preliminary experiences. Acta Radiol Onco Radiat Phys Biol 1978;17:150-60.

[26] Bouchet A, Lemasson B, Leduc G, Maisin C, Bräuer-Krisch E, Siegbahn AE, et al. Preferential effect of synchrotron microbeam radiation therapy on intracerebral 9L gliosarcoma vascular networks. Int J Radiat Oncol Biol Phys 2010;78: $1503-12$

[27] Griffin RJ, Koonce NA, Dings RPM, Siegel E, Moros EG, Bräuer-Krisch E, et al. Microbeam radiation therapy alters vascular architecture and tumor oxygenation and is enhanced by a galectin- 1 targeted anti-angiogenic peptide. Radiat Res 2012;177:804-12.

[28] Smilowitz HM, Blattmann H, Bräuer-Krisch E, Bravin A, Michiel MD, Gebbers JO, et al. Synergy of gene-mediated immunoprophylaxis and microbeam radiation therapy for advanced intracerebral rat 9L gliosarcomas. J Neurooncol 2006;78:135-43.

[29] Park HJ, Griffin RJ, Hui S, Levitt SH, Song CW. Radiation-induced vascular damage in tumors: implications of vascular damage in ablative hypofractionated radiotherapy (SBRT and SRS). Radiat Res 2012;177:311-27.

[30] Bouchet A, Lemasson B, Christen T, Potez M, Rome C, Coquery N, et al. Synchrotron microbeam radiation therapy induces hypoxia in intracerebral gliosarcoma but not in the normal brain. Radiother Oncol 2013;108:143-8.

[31] Laissue JA, Bartzsch S, Blattmann H, Bräuer-Krisch E, Bravin A, Dalléry D, et al Response of the rat spinal cord to X-ray microbeams. Radiother Oncol 2013;106:106-11.

[32] Kolesnick R, Fuks Z. Radiation and ceramide-induced apoptosis. Oncogene 2003;22:5897-906.

[33] Smith RW, Wang J, Schültke E, Seymour CB, Bräuer-Krisch E, Laissue JA, et al. Proteomic changes in the rat brain induced by homogenous irradiation and by the bystander effect resulting from high energy synchrotron X-ray microbeams. Int J Radiat Biol 2013;89:118-27.

[34] Bouchet A, Sakakini N, El Atifi M, Le Clec'h C, Bräuer-Krisch E, Rogalev L, et al. Identification of AREG and PLK1 pathway modulation as a potential key of the response of intracranial 9L tumor to microbeam radiation therapy. Int J Cancer 2015 Jun 1;136(11):2705-16.

[35] Bouchet A, Sakakini N, El Atifi M, Le Clec'h C, Brauer E, Moisan A, et al. Early gene expression analysis in 9L orthotopic tumor-bearing rats identifies immune modulation in molecular response to synchrotron microbeam radiation therapy. PLoS One 2013;8:e81874.

[36] Degenhardt Y, Lampkin T. Targeting polo-like kinase in cancer therapy. Clin Cancer Res 2010;16:384-9.

[37] Crosbie JC, Anderson RL, Rothkamm K, Restall CM, Cann L, Ruwanpura S, et al. Tumor cell response to synchrotron microbeam radiation therapy differs markedly from cells in normal tissues. Int J Radiat Oncol Biol Phys 2010;77: 886-94.

[38] Toulany M, Dittmann K, Krüger M, Baumann M, Rodemann HP. Radioresistance of K-Ras mutated human tumor cells is mediated through EGFRdependent activation of PI3K-AKT pathway. Radiother Oncol 2005;76: 143-50.

[39] Sprung CN, Yang Y, Forrester HB, Li J, Zaitseva M, Cann L, et al. Genome-wide transcription responses to synchrotron microbeam radiotherapy. Radiat Res 2012;178:249-59.

[40] Brandes AA, Franceschi E, Tosoni A, Hegi ME, Stupp R. Epidermal growth factor receptor inhibitors in neuro-oncology: hopes and disappointments. Clin Cancer Res 2008;14:957-60. 